\title{
Quantification of Uncertainty in the Mathematical Modelling of a Multivariable Suspension Strut Using Bayesian Interval Hypothesis-Based Approach
}

\author{
Shashidhar Mallapur ${ }^{1}$ and Roland Platz ${ }^{2}$ \\ ${ }^{1}$ Technische Universität Darmstadt, System Reliability, Adaptive Structures and Machine Acoustics \\ SAM, Magdalenenstraße 4, 64289 Darmstadt, Germany \\ ${ }^{2}$ Fraunhofer Institute for Structural Durability and System Reliability LBF, \\ Bartningstraße 47, 64289 Darmstadt, Germany \\ ${ }^{1}$ mallapur@sam.tu-darmstadt.de, ${ }^{2}$ roland.platz@lbf.fraunhofer.de
}

\begin{abstract}
Keywords: Bayesian interval hypothesis, mathematical model, model validation, marginal likelihood, suspension strut, uncertainty
\end{abstract}

\begin{abstract}
Mathematical models of a suspension strut such as an aircraft landing gear are utilized by engineers in order to predict its dynamic response under different boundary conditions. The prediction of the dynamic response, for example the external loads, the stress and the strength as well as the maximum compression in the spring-damper component aids engineers in early decision making to ensure its structural reliability under various operational conditions. However, the prediction of the dynamic response is influenced by model uncertainty. As far as the model uncertainty is concerned, the prediction of the dynamic behavior via different mathematical models depends upon various factors such as the model's complexity in terms of the degrees of freedom, material and geometrical assumptions, their boundary conditions and the governing functional relations between the model input and output parameters. The latter can be linear or nonlinear, axiomatic or empiric, time variant or timeinvariant. Hence, the uncertainty that arises in the prediction of the dynamic response of the resulting different mathematical models needs to be quantified with suitable validation metrics, especially when the system is under structural risk and failure assessment. In this contribution, the authors utilize the Bayesian interval hypothesis-based method to quantify the uncertainty in the mathematical models of the suspension strut.
\end{abstract}

\section{Introduction}

Since the mid-1960s, mathematical simulation models have been used as a tool in the field of scientific research as well as in the design and development of engineering systems [1]. Mathematical models are used to predict the non-deterministic dynamic response of engineering systems under various static and kinetic loading conditions [2]. The predicted responses are used by engineers to evaluate the system's stability, strength and vibrational behaviour under various operational conditions. However, the mathematical models are influenced by the uncertainty in the assumed model parameters, the initial and boundary conditions, the input variables as well as the governing functional relations between the model input and output variables [3]. Hence, the resulting mathematical models that predict the dynamic responses differently need to be assessed with suitable validation metrics [4], [5], [6]. A validation metric gives a quantitative measure of agreement between the predicted response and the experimentally observed response [7], [8]. The validation metric must take into account the uncertainty in the mathematical models as well as in the experimental observations of the dynamic response of the engineering system in interest [9].

The validation metrics are used for model selection when alternative mathematical models are being considered. Various validation metrics such as the classical hypothesis testing, Bayes factor, frequentist's metric and area metric are discussed in [10] and [11]. As for the probabilistic methods, validation metrics such as BAYES factor which is based on BAYESIAN probabilistic approach can be 
utilized to judge the capability of prediction of a given mathematical model with the experimental data [12]. Model validation is classified as either univariate or multivariate. Bayesian validation metrics for univariate problems have been discussed in [13], [14]. However, for multivariate engineering systems under uncertainty different validation metrics based on the Bayesian hypothesis appproach and the area metric approach are discussed in [15], [16]. Data uncertainty occurs when the input parameters, such as state variables or the system parameters of the mathematical models are uncertain. This can be represented using probabilistic and non-probabilistic approaches, [17], [18] and [19]. The sources of uncertainty can be broadly classified as aleatoric or epistemic [10].

In this contribution, the authors consider the BAYESIAN interval hypothesis as a model validation metric according to [20] to quantify the uncertainty in the mathematical modeling of a suspension strut. However, and as a new approach, the authors present a way to adequately compare different models based on linear, nonlinear, axiomatic or empiric assumptions of functional relations. This work considers a suspension strut which is referred as the modular active spring-damper system (German acronym MAFDS). MAFDS is developed under the framework of German Collaborative Research Center SFB 805 at the Technische Universität Darmstadt. The concept of MAFDS is registered as a Patent DE 102014 106 858.A1 "Load transferring device” [21].

\section{Modular Active Spring Damper System MAFDS}

Fig. 1 (a) illustrates the MAFDS. The MAFDS serves as an academic demonstrator to investigate the different methods and technologies to control uncertainty in all development stages such as the conceptual phase, design and optimization phase, production and assembly phase as well as the final operational phase. The MAFDS has similar dynamic requirements of a typical suspension strut such as an aircraft landing gear. However, it is not designed to be used as a substitute to an existing aircraft landing gear. Rather, the MAFDS is an academic example to study the uncertainty in the dynamic outputs of suspension strut systems when different passive, semi-active and active modules are used to lower the uncertainty and to enhance the performance. It carries a payload on an upper truss that is connected to the lower truss via guidance links. The guidance link enables the kinematic motion between the lower and upper truss. The elastic foot acts as an impact absorption element which introduces the axial and lateral forces due to impact into the MAFDS.

The axial forces are transferred to the upper truss via the spring-damper component that is loaded by axial loads only due to load redirection in the upper truss of the MAFDS, Fig. 1 (b). The load redirection in the upper truss is mentioned as a key function in the patent of MAFDS "Load transferring device" [21]. An impact scenario is created by releasing the MAFDS from a specified drop height $h$. Uncertainty is investigated especially for MAFDS's main operating function and purpose: sustain stability, well balanced load distribution and ability to attenuate vibrations. Passive, semi-active and active technology approaches for stability, load distribution and vibration control are developed to compensate uncertainty in the main functions mentioned above. They are integrated in a modular way within the SFB 805 [26], [27], [28], [19], [22], [23], [24] and [25].

In this contribution, the global dynamic outputs of the MAFDS are the relative compression and the forces exerted by the spring damper component, and the ground impact force at the elastic foot under drop test scenario. For this, uncertainty as the varying payload and varying drop heights are assumed numerically via different mathematical models subject to experimental validation via test rig. 


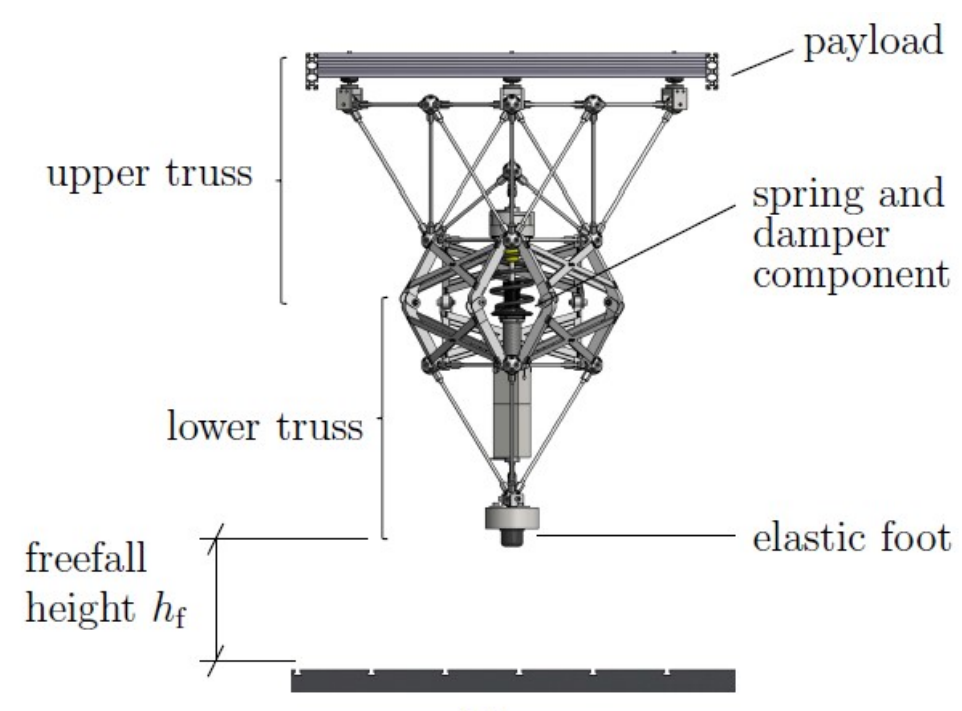

(a)

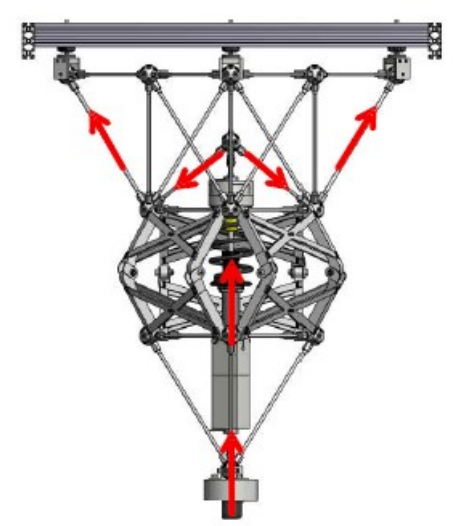

(b)

Fig. 1: (a) CAD illustration of suspension strut MAFDS, (b) Load redirection in upper truss of MAFDS

\section{Simple Mathematical Modelling of MAFDS}

\subsection{Motivation}

Fig. 2 shows a simplified two degree of freedom (2DOF) mathematical model of the MAFDS. It is utilized to predict the global dynamic output of the spring damper component and the ground impact force of MAFDS under varying drop heights and payloads. The internal loads in the upper and lower trusses are neglected. The upper and lower structures of the MAFDS are simplified as two lumped upper mass $m_{\mathrm{u}}$ and lower mass $m_{1}$. The spring-damper component is represented by the stiffness parameter $k$ and the viscous damping coefficient $b$. Similarly, the elastic foot is represented by the stiffness parameter $k_{\text {ef }}$. The 2DOF mathematical model has two degrees of freedom that are defined by the local translational displacements $z_{\mathrm{u}}$ and $z_{1}$.

The 2DOF mathematical model in Fig. 2 is assumed to be under free and full homogeneous field of acceleration due to gravity $g$. The model is raised to the drop height $h$ for the free fall drop test in order to excite the 2DOF-model in Fig. 2. Depending upon the drop height $h$, the model is excited by the initial conditions that are applied to $m_{\mathfrak{u}}$ and $m_{1}$. Thus the initial displacements $z_{0}$ and the initial velocities $\dot{z}_{0}$ at time $t=0$ are

$$
z_{0}=0, \quad \dot{z}_{0}=0,
$$

and hence the velocity $\dot{z}(h)$ due to drop height $h$ becomes

$$
\dot{z}(h)=-\sqrt{2 g h} .
$$

The goal is to predict the maximum dynamic response vector

$$
\boldsymbol{y}_{\mathrm{m}, \max }=\left[\begin{array}{c}
z_{\mathrm{r}, \mathrm{m}, \max } \\
F_{\mathrm{sd}, \mathrm{m}, \max } \\
F_{\mathrm{ef}, \mathrm{m}, \max }
\end{array}\right]=\max \left[\begin{array}{c}
z_{\mathrm{u}}-z_{1} \\
k\left(z_{\mathrm{u}}-z_{1}\right)+b\left(\dot{z}_{\mathrm{u}}-\dot{z}_{1}\right) \\
k_{\mathrm{ef}} z_{1}
\end{array}\right]
$$

that is related to the displacements $z_{\mathrm{u}}, z_{1}$ and the velocities $\dot{z}_{\mathrm{u}}, \dot{z}_{1}$. It consists of the maximum modeled compression $z_{\mathrm{r}, \mathrm{m}, \max }$, maximum spring-damper force $F_{\mathrm{sd}, \mathrm{m}, \max }$ and the maximum ground impact force $F_{\text {ef,m,max }}$. 


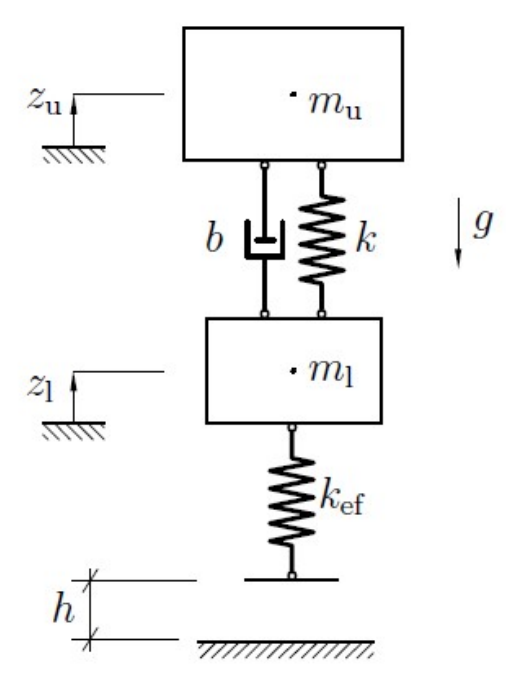

(a)

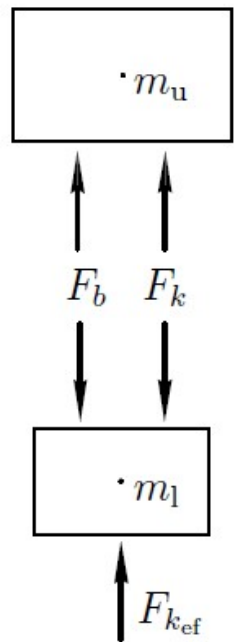

(b)

Fig. 2: Illustration of simplified 2DOF mathematical model of MAFDS: a) principal sketch and b) internal free forces

\subsection{Four Different Approaches to Model the Stiffness and the Damping}

The 2DOF mathematical model can be described with four underlying assumptions with respect to the model parameter's functional relations: linear or nonlinear, axiomatic or empiric. The authors present four different approaches to model the stiffness and the damping of the 2DOF mathematical model for the prediction of the dynamic response $\boldsymbol{y}$ vector of the 2DOF model.

\section{a) Model with linear axiomatic stiffness $k$ and linear axiomatic damping $b$}

According to Fig. 2 (b), the free body diagram forces are

$$
F_{b}=b\left[\dot{z}_{\mathrm{u}}-\dot{z}_{1}\right], \quad F_{k}=k\left[z_{\mathrm{u}}-z_{1}\right], \quad F_{k \mathrm{ef}}=k_{\mathrm{ef}} z_{1}
$$

with the linear axiomatic viscous damping force $F_{b}$, the linear axiomatic spring force $F_{k}$ and the force $F_{k \text { ef }}$ exerted via the elastic foot. The governing homogeneous differential equation of the 2DOF mathematical model for $m_{\mathrm{u}}$ is

$$
m_{\mathrm{u}} \ddot{z}_{\mathrm{u}}+b\left[\dot{z}_{\mathrm{u}}-\dot{z}_{1}\right]+k\left[z_{\mathrm{u}}-z_{1}\right]=0
$$

and for $m_{1}$

$$
m_{1} \ddot{z}_{1}-b\left[\dot{z}_{\mathrm{u}}-\dot{z}_{1}\right]-k\left[z_{\mathrm{u}}-z_{1}\right]+k_{\mathrm{ef}} z_{1}=0 .
$$

The matrix form representation of (5) and (6) is expressed as

$$
\underbrace{\left[\begin{array}{cc}
m_{\mathrm{u}} & 0 \\
0 & m_{1}
\end{array}\right]}_{\boldsymbol{M}}\left(\begin{array}{l}
\ddot{z}_{\mathrm{u}} \\
\ddot{z}_{1}
\end{array}\right)+\underbrace{\left[\begin{array}{cc}
b & -b \\
-b & b
\end{array}\right]}_{\boldsymbol{B}}\left(\begin{array}{c}
\dot{z}_{\mathrm{u}} \\
\dot{z}_{1}
\end{array}\right)+\underbrace{\left[\begin{array}{cc}
k & -k \\
-k & k+k_{\mathrm{ef}}
\end{array}\right]}_{\boldsymbol{K}}\left(\begin{array}{l}
z_{\mathrm{u}} \\
z_{1}
\end{array}\right)=\left(\begin{array}{l}
0 \\
0
\end{array}\right),
$$

with the global mass, damping and stiffness matrices $M, B$ and $K$. 


\section{b) Model with nonlinear empiric stiffness $k\left(z_{\mathrm{r}}\right)$ and linear axiomatic damping $b$}

In this mathematical model, the stiffness $k\left(z_{\mathrm{r}}\right)$ of the spring is not constant and is modelled as a function that is dependent upon the value of the relative displacement $z_{\mathrm{r}}=\left(z_{\mathrm{u}}-z_{1}\right)$. The empiric stiffness data points in Fig. 3 are obtained from the experimental static relative displacement under varying static loads that is conducted via the MAFDS test rig. The relative displacement $z_{\mathrm{r}}$ increases nonlinearly for $z_{\mathrm{r}}>0.07 \mathrm{~m}$. The spring force is modelled as

$$
F_{k\left(z_{\mathrm{r}}\right)}=k\left(z_{\mathrm{r}}\right)\left[z_{\mathrm{u}}-z_{1}\right]
$$

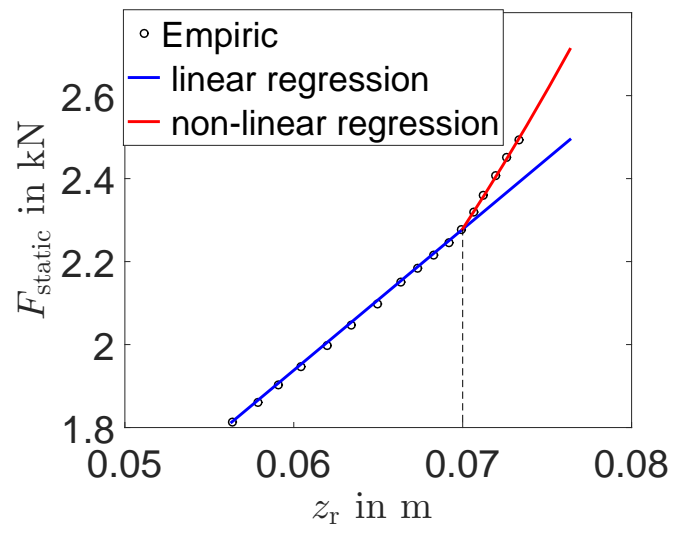

Fig. 3: Stiffness curve

with the nonlinearity described with a regression function

$$
k\left(z_{\mathrm{r}}\right)=\left\{\begin{array}{l}
k_{1}=3.3 \cdot 10^{4} \mathrm{Nm}^{-1}, \text { for } 0.056 \mathrm{~m} \leq z_{\mathrm{r}} \leq 0.07 \mathrm{~m} \\
k_{2}\left(z_{\mathrm{r}}\right)=k_{2,2} z_{\mathrm{r}}^{2}-k_{2,1} z_{\mathrm{r}}+k_{2,0}, \text { for } 0.07 \mathrm{~m}<z_{\mathrm{r}} \leq 0.075 \mathrm{~m}
\end{array}\right.
$$

and with the cubic stiffness parameter $k_{2,2}=6.5 \cdot 10^{6} \mathrm{Nm}^{-3}$, quadratic stiffness parameter $k_{2,1}=4.9 \cdot 10^{5} \mathrm{Nm}^{-2}$ and linear stiffness $k_{2,0}=3.3 \cdot 10^{4} \mathrm{Nm}^{-1}$. As seen in Fig. 3, the nonlinear regression has only a minor slope in $k_{2}\left(z_{\mathrm{r}}\right)$ that is small compared to the linear regression assumption for $k_{1}$. The matrix form representation of the homogeneous governing differential equation is expressed as

$$
\underbrace{\left[\begin{array}{cc}
m_{\mathrm{u}} & 0 \\
0 & m_{1}
\end{array}\right]}_{\boldsymbol{M}}\left(\begin{array}{l}
\ddot{z}_{\mathrm{u}} \\
\ddot{z}_{1}
\end{array}\right)+\underbrace{\left[\begin{array}{cc}
b & -b \\
-b & b
\end{array}\right]}_{\boldsymbol{B}}\left(\begin{array}{l}
\dot{z}_{\mathrm{u}} \\
\dot{z}_{1}
\end{array}\right)+\underbrace{\left[\begin{array}{cc}
k\left(z_{\mathrm{r}}\right) & -k\left(z_{\mathrm{r}}\right) \\
-k\left(z_{\mathrm{r}}\right) & k\left(z_{\mathrm{r}}\right)+k_{\mathrm{ef}}
\end{array}\right]}_{\boldsymbol{K}\left(\boldsymbol{z}_{\mathbf{r}}\right)}\left(\begin{array}{c}
z_{\mathrm{u}} \\
z_{1}
\end{array}\right)=\left(\begin{array}{l}
0 \\
0
\end{array}\right) .
$$

c) Model with linear axiomatic stiffness $k$ and nonlinear empiric damping $b \propto F_{b}\left(\dot{z}_{\mathrm{r}}(t)\right)$

The damping forces $F_{b}$ in the mathematical models a) and b) are based on linear assumptions. The damping force $F_{b}\left(\dot{z}_{\mathrm{r}}\right)$ is obtained via experimental drop tests of MAFDS. It is seen that the empiric damping force $F_{b}\left(\dot{z}_{\mathrm{r}}\right)$ that is obtained from the experimental test of MAFDS is related to the relative

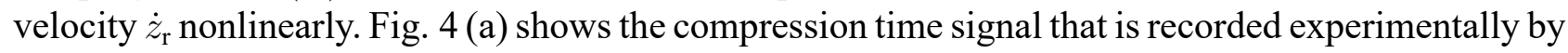
drop tests. The noise in the compression signal was filtered with a lowpass BUTTERWORTH filter. The relative velocity $\dot{z}_{\mathrm{r}}$ shown in Fig. 4 (b) is calculated from the measured compression $z_{\mathrm{r}}$ via numerical differentiation over time. Eventually, $F_{b}\left(\dot{z}_{\mathrm{r}}\right)$ is seen in Fig. 4 (c) as a function of $\dot{z}_{\mathrm{r}}$.

The empiric damping force $F_{b}\left(\dot{z}_{\mathrm{r}}\right)$ can be modeled as

$$
F_{b}\left(\dot{z}_{\mathrm{r}}\right)=b\left(\dot{z}_{\mathrm{r}}\right)\left[\dot{z}_{\mathrm{u}}-\dot{z}_{1}\right]
$$

with the nonlinear damping behavior described by an adapted regression function

$$
b\left(\dot{z}_{\mathrm{r}}\right)=b_{3} \dot{z}_{\mathrm{r}}^{2}-b_{2} \dot{z}_{\mathrm{r}}+b_{1}
$$

with the cubic damping coefficient $b_{3}=1.3 \cdot 10^{3} \mathrm{Ns} \mathrm{m}^{-3}$, quadratic damping coefficient $b_{2}=4.1 \cdot 10^{3} \mathrm{Nsm}^{-2}$ and linear damping coefficient $b_{1}=4.7 \cdot 10^{3} \mathrm{Ns} \mathrm{m}^{-1}$. The matrix form of the homogeneous governing differential equation is 


$$
\underbrace{\left[\begin{array}{cc}
m_{\mathrm{u}} & 0 \\
0 & m_{1}
\end{array}\right]}_{\boldsymbol{M}}\left(\begin{array}{l}
\ddot{z}_{\mathrm{u}} \\
\ddot{z}_{1}
\end{array}\right)+\underbrace{\left[\begin{array}{cc}
b\left(\dot{z}_{\mathrm{r}}\right) & -b\left(\dot{z}_{\mathrm{r}}\right) \\
-b\left(\dot{z}_{\mathrm{r}}\right) & b\left(\dot{z}_{\mathrm{r}}\right)
\end{array}\right]}_{\boldsymbol{B}\left(\dot{\boldsymbol{z}}_{\mathrm{r}}\right)}\left(\begin{array}{c}
\dot{z}_{\mathrm{u}} \\
\dot{z}_{1}
\end{array}\right)+\underbrace{\left[\begin{array}{cc}
k & -k \\
-k & k+k_{\mathrm{ef}}
\end{array}\right]}_{\boldsymbol{K}}\left(\begin{array}{c}
z_{\mathrm{u}} \\
z_{1}
\end{array}\right)=\left(\begin{array}{l}
0 \\
0
\end{array}\right) .
$$

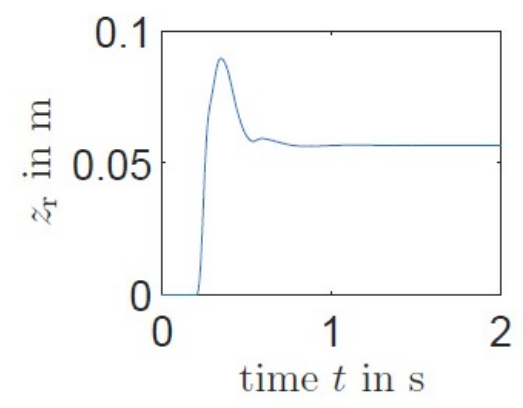

(a)

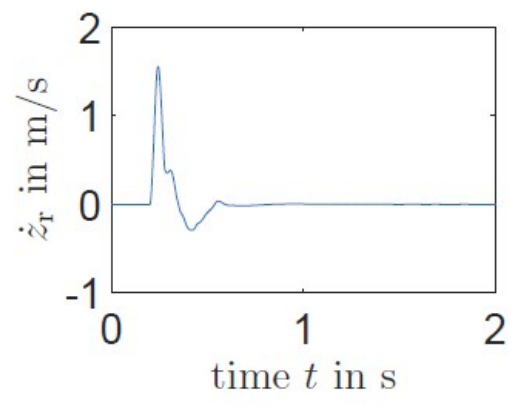

(b)

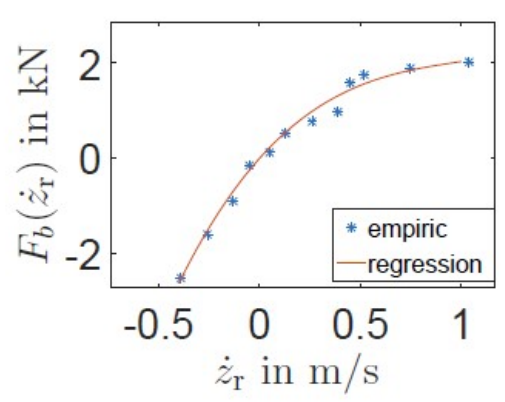

(c)

Fig. 4: a) relative displacement $z_{\mathrm{r}}$, b) relative velocity $\dot{z}_{\mathrm{r}}$ and c) velocity dependent nonlinear damping force $F_{b}\left(\dot{z}_{\mathrm{r}}\right)$

d) Model with nonlinear empiric stiffness $k\left(z_{\mathrm{r}}\right)$ and nonlinear empiric damping $b \propto F_{b}\left(\dot{z}_{\mathrm{r}}(t)\right)$

In this mathematical model, the assumptions about the stiffness behavior in model b) and the damping behavior in model c) are combined. The resulting governing relation in the matrix form is

$$
\underbrace{\left[\begin{array}{cc}
m_{\mathrm{u}} & 0 \\
0 & m_{1}
\end{array}\right]}_{\boldsymbol{M}}\left(\begin{array}{l}
\ddot{z}_{\mathrm{u}} \\
\ddot{z}_{1}
\end{array}\right)+\underbrace{\left[\begin{array}{cc}
b\left(\dot{z}_{\mathrm{r}}\right) & -b\left(\dot{z}_{\mathrm{r}}\right) \\
-b\left(\dot{z}_{\mathrm{r}}\right) & b\left(\dot{z}_{\mathrm{r}}\right)
\end{array}\right]}_{\boldsymbol{B}\left(\dot{\boldsymbol{z}}_{\mathrm{r}}\right)}\left(\begin{array}{c}
\dot{z}_{\mathrm{u}} \\
\dot{z}_{1}
\end{array}\right)+\underbrace{\left[\begin{array}{cc}
k\left(z_{\mathrm{r}}\right) & -k\left(z_{\mathrm{r}}\right) \\
-k\left(z_{\mathrm{r}}\right) & k\left(z_{\mathrm{r}}\right)+k_{\mathrm{ef}}
\end{array}\right]}_{\boldsymbol{K}\left(\boldsymbol{z}_{\mathrm{r}}\right)}\left(\begin{array}{c}
z_{\mathrm{u}} \\
z_{1}
\end{array}\right)=\left(\begin{array}{l}
0 \\
0
\end{array}\right) .
$$

\subsection{Solution of the Relative Compression Response of the 2DOF Mathematical Models a) to d)}

The solution in time domain for (7), (10), (13) and (14) is described in a form that leads to the dynamic response $\boldsymbol{y}_{\mathrm{m}, \max }$ in (3). It is to be noted that $\boldsymbol{y}_{\mathrm{m}, \max }$ is applicable for the different linear, nonlinear, axiomatic and empiric assumptions for stiffness and damping behaviour. The solutions are obtained by numerical integration via RUNGE KuTTA algorithm. From (5) and (6), the accelerations $\ddot{z}_{\mathrm{u}}$ and $\ddot{z}_{1}$ are

$$
\ddot{z}_{\mathrm{u}}=\frac{1}{m_{\mathrm{u}}}\left[-b\left[\dot{z}_{\mathrm{u}}-\dot{z}_{1}\right]-k\left[z_{\mathrm{u}}-z_{1}\right]\right],
$$

and

$$
\ddot{z}_{1}=\frac{1}{m_{1}}\left[b\left[\dot{z}_{\mathrm{u}}-\dot{z}_{1}\right]+k\left[z_{\mathrm{u}}-z_{1}\right]-k_{\mathrm{ef}} z_{1}\right],
$$

which leads to the state-space form of (15) and (16)

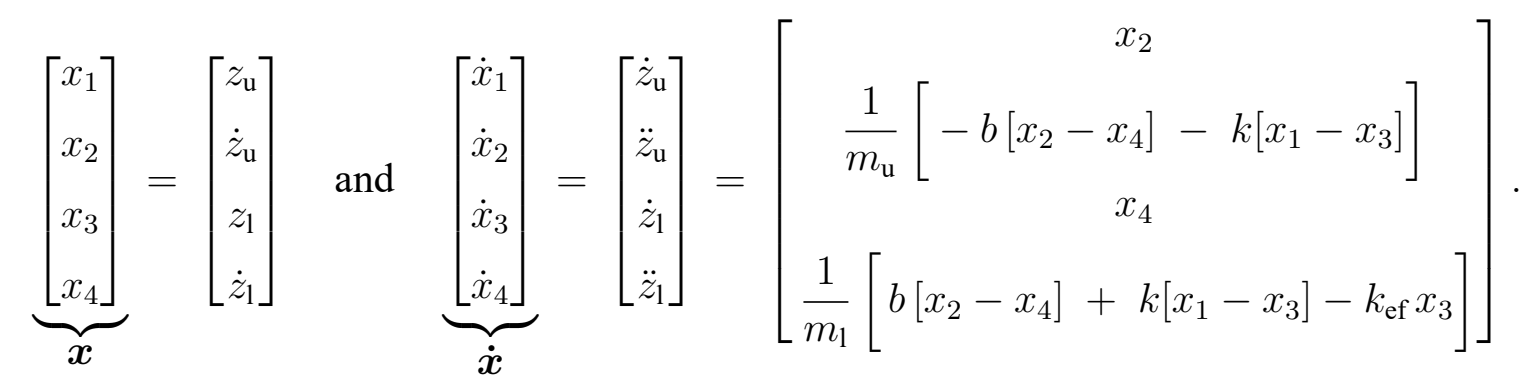


Similarly, the general time domain solution to the governing relations for linear, nonlinear, axiomatic and empiric assumptions in (7), (10), (13) and (14) can be expressed in a generalized nonlinear state space form by

$$
\dot{\boldsymbol{x}}=\left[\begin{array}{c}
x_{2} \\
\frac{1}{m_{\mathrm{u}}} f_{\mathrm{u}}\left(x_{1}, x_{2}, x_{3}, x_{4}\right) \\
x_{4} \\
\frac{1}{m_{1}} f_{1}\left(x_{1}, x_{2}, x_{3}, x_{4}\right)
\end{array}\right] .
$$

The summation of internal forces acting on $m_{\mathrm{u}}$ and $m_{1}$ in (18) are described as internal force functions $f_{\mathrm{u}}\left(x_{1}, x_{2}, x_{3}, x_{4}\right)$ and $f_{1}\left(x_{1}, x_{2}, x_{3}, x_{4}\right)$, where $x_{1}, \ldots, x_{4}$ are the state variables that represent the displacements and the velocities in (17). The functions $f_{\mathrm{u}}\left(x_{1}, x_{2}, x_{3}, x_{4}\right)$ and $f_{1}\left(x_{1}, x_{2}, x_{3}, x_{4}\right)$ for the mathematical models a) to d) are listed in Tab. 1 . The state vector $\dot{\boldsymbol{x}}$ is numerically integrated using a fourth order RUNGE KUTTA algorithm to obtain the desired state variables $x_{1}, x_{2}, x_{3}$ and $x_{4}$. The numerical computation is started with the initial conditions in (1) and (2) along with the numerical time integration step $t_{\mathrm{s}}=0.001 \mathrm{~s}$. Hence, the general desired dynamic output becomes

$$
\boldsymbol{y}_{\mathrm{m}}=\left[\begin{array}{c}
z_{\mathrm{r}} \\
F_{\mathrm{sd}} \\
F_{\mathrm{ef}}
\end{array}\right]=\left[\begin{array}{cccc}
1 & 0 & -1 & 0 \\
k & b & -k & -b \\
0 & 0 & -k_{\mathrm{ef}} & 0
\end{array}\right]\left[\begin{array}{c}
z_{\mathrm{u}} \\
\dot{z}_{\mathrm{u}} \\
z_{1} \\
\dot{z}_{1}
\end{array}\right]=\left[\begin{array}{cccc}
1 & 0 & -1 & 0 \\
k & b & -k & -b \\
0 & 0 & -k_{\mathrm{ef}} & 0
\end{array}\right] \underbrace{\left[\begin{array}{c}
x_{1} \\
x_{2} \\
x_{3} \\
x_{4}
\end{array}\right]}_{\boldsymbol{x}} .
$$

Tab. 2 summarizes the model parameters for the mathematical models a) to d). According to Fig. 1 (a), $m_{\mathrm{u}}$ and $m_{1}$ are the masses of the upper and lower structure, $k, k\left(z_{r}\right)$ and $k_{\text {ef }}$ are the stiffnesses for linear, nonlinear displacement dependent and elastic foot property assumptions, $b$ and $b\left(\dot{z}_{r}\right)$ are the viscous and velocity dependent nonlinear damping coefficients, $h$ is the drop height. The values of the displacement dependent nonlinear stiffness and of the velocity dependent nonlinear damping are valid within the given intervals in Tab. 2. The model parameters that are taken into account in the models a) to d) are designated with $(\checkmark)$, otherwise with $(\times)$.

Table 1: Functions $f_{\mathrm{u}}\left(x_{1}, x_{2}, x_{3}, x_{4}\right)$ and $f_{1}\left(x_{1}, x_{2}, x_{3}, x_{4}\right)$ for different mathematical models

\begin{tabular}{ll}
\hline \hline model type & functions $f_{\mathrm{u}}\left(x_{1}, x_{2}, x_{3}, x_{4}\right)$ and $f_{1}\left(x_{1}, x_{2}, x_{3}, x_{4}\right)$ \\
\hline \hline model a) & $f_{\mathrm{u}}\left(x_{1}, x_{2}, x_{3}, x_{4}\right)=-b\left[x_{2}-x_{4}\right]-k\left[x_{1}-x_{3}\right]$ \\
& $f_{1}\left(x_{1}, x_{2}, x_{3}, x_{4}\right)=b\left[x_{2}-x_{4}\right]+k\left[x_{1}-x_{3}\right]-k_{\mathrm{ef}} x_{3}$ \\
\hline model $\mathrm{b})$ & $f_{\mathrm{u}}\left(x_{1}, x_{2}, x_{3}, x_{4}\right)=-b\left[x_{2}-x_{4}\right]-k\left(z_{\mathrm{r}}\right)\left[x_{1}-x_{3}\right]$ \\
& $f_{1}\left(x_{1}, x_{2}, x_{3}, x_{4}\right)=b\left[x_{2}-x_{4}\right]+k\left(z_{\mathrm{r}}\right)\left[x_{1}-x_{3}\right]-k_{\mathrm{ef}} x_{3}$ \\
\hline model c) & $f_{\mathrm{u}}\left(x_{1}, x_{2}, x_{3}, x_{4}\right)=-b\left(\dot{z}_{\mathrm{r}}\right)\left[x_{2}-x_{4}\right]-k\left[x_{1}-x_{3}\right]$ \\
& $f_{1}\left(x_{1}, x_{2}, x_{3}, x_{4}\right)=b\left(\dot{z}_{\mathrm{r}}\right)\left[x_{2}-x_{4}\right]+k\left[x_{1}-x_{3}\right]-k_{\mathrm{ef}} x_{3}$ \\
\hline model d) & $f_{\mathrm{u}}\left(x_{1}, x_{2}, x_{3}, x_{4}\right)=-b\left(\dot{z}_{\mathrm{r}}\right)\left[x_{2}-x_{4}\right]-k\left(z_{\mathrm{r}}\right)\left[x_{1}-x_{3}\right]$ \\
& $f_{1}\left(x_{1}, x_{2}, x_{3}, x_{4}\right)=b\left(\dot{z}_{\mathrm{r}}\right)\left[x_{2}-x_{4}\right]+k\left(z_{\mathrm{r}}\right)\left[x_{1}-x_{3}\right]-k_{\mathrm{ef}} x_{3}$ \\
\hline \hline
\end{tabular}


Table 2: Model parameters used for different mathematical models

\begin{tabular}{llllllll}
\hline \hline parameter & symbol & value & SI unit & a) & b) & c) & d) \\
\hline mass of upper structure & $m_{\mathrm{u}}$ & 185 & $\mathrm{~kg}$ & $\checkmark$ & $\checkmark$ & $\checkmark$ & $\checkmark$ \\
mass of lower structure & $m_{\mathrm{l}}$ & 41 & $\mathrm{~kg}$ & $\checkmark$ & $\checkmark$ & $\checkmark$ & $\checkmark$ \\
linear stiffness & $k$ & $33 \cdot 10^{3}$ & $\mathrm{~N} / \mathrm{m}$ & $\checkmark$ & $\times$ & $\checkmark$ & $\times$ \\
nonlinear stiffness & $k\left(z_{\mathrm{r}}\right)$ & {$\left[33 \cdot 10^{3}, \ldots, 39.5 \cdot 10^{3}\right]$} & $\mathrm{N} / \mathrm{m}$ & $\times$ & $\checkmark$ & $\times$ & $\checkmark$ \\
linear elastic foot stiffness & $k_{\mathrm{ef}}$ & $224 \cdot 10^{3}$ & $\mathrm{~N} / \mathrm{m}$ & $\checkmark$ & $\checkmark$ & $\checkmark$ & $\checkmark$ \\
linear damping & $b$ & $1.8 \cdot 10^{3}$ & $\mathrm{Ns} / \mathrm{m}$ & $\checkmark$ & $\checkmark$ & $\times$ & $\times$ \\
nonlinear damping & $b\left(\dot{z}_{\mathrm{r}}\right)$ & {$\left[2.0 \cdot 10^{3}, \ldots, 4.9 \cdot 10^{3}\right]$} & $\mathrm{Ns} / \mathrm{m}$ & $\times$ & $\times$ & $\checkmark$ & $\checkmark$ \\
drop height & $h$ & {$[0.03,0.04,0.05]$} & $\mathrm{m}$ & $\checkmark$ & $\checkmark$ & $\checkmark$ & $\checkmark$ \\
\hline
\end{tabular}

\section{Experimental Test Rig of MAFDS}

Fig. 5 describes the experimental test rig of the MAFDS that is compared with the four simple 2DOF mathematical models a) to d) with respect to measured versus numerically predicted outputs by drop tests, Tab. 3 summarizes the test rig's components. The MAFDS is fixed at three points to the load frame 1 by means of three bearing supports 2 . The hoist frame 3 and the load frame 1 are connected by an electro-pneumatic coupling 4. The hoist and the load frames are fixed to the guide blocks 5 that allow smooth parallel movement of the MAFDS along the guide rails 6 . The hoist frame is driven by two servomotors 7 that run synchronously. The ball screw 8 converts the rotational motion of the threaded spindle 9 , which is powered by the servomotors into the translational parallel movement of the hoist frame. Once lifted to the desired experimental drop height $h$, the MAFDS is released by a pneumatic coupling for a drop test. The maximum experimental drop height of MAFDS is $h_{\max }=0.22 \mathrm{~m}$. Additional payloads $10 \mathrm{can}$ be fixed to the load frame, thereby adding static loads to the MAFDS.

The dynamic response vector $\boldsymbol{y}_{\mathrm{m}, \max }$ in (3) predicted by the mathematical models is validated by conducting experimental drop tests to get the experimental dynamic response vector

$$
\boldsymbol{y}_{\mathrm{e}, \max }=\left[\begin{array}{c}
z_{\mathrm{r}, \mathrm{e}, \max } \\
F_{\mathrm{sd}, \mathrm{e}, \max } \\
F_{\mathrm{ef}, \mathrm{e}, \max }
\end{array}\right]
$$

with multi variable inputs such as the experimental mass of the upper structure $m_{\mathfrak{u}}$ and the experimental drop height $h$. The measured dynamic output variable in (20) comprises of the maximum compression $z_{\mathrm{r}, \mathrm{e} \text { max }}$, maximum spring damper force $F_{\mathrm{sd}, \text { e,max }}$ and the maximum ground impact force $F_{\text {ef,e,max }}$. Fig. 6 illustrates the MAFDS and the experimental test rig perspectively. 


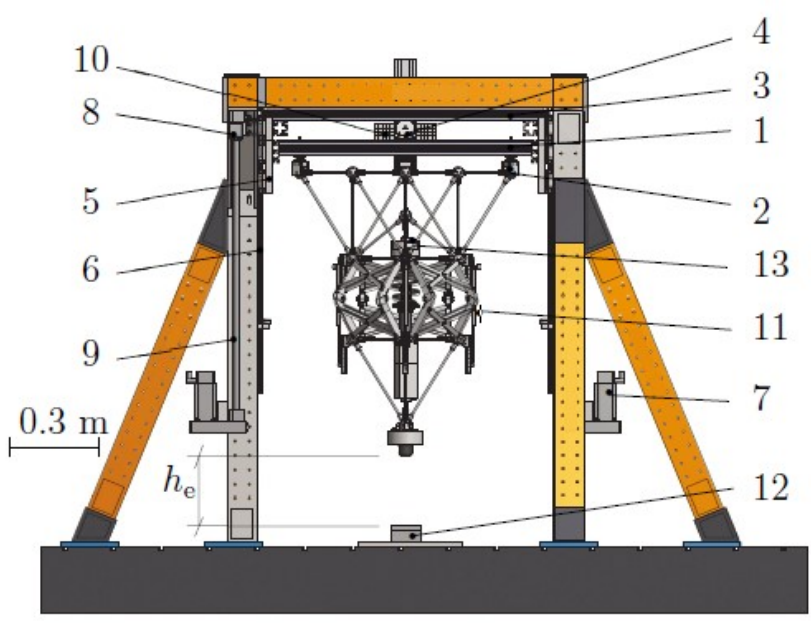

(a)

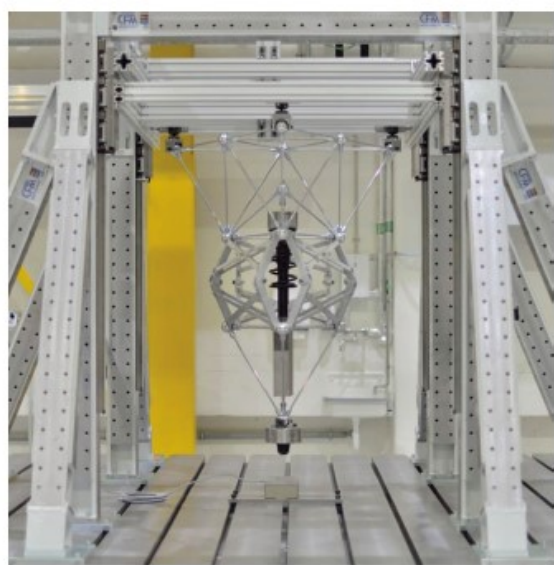

(b)

Fig. 5: Experimental rig for MAFDS, (a) CAD illustration of experimental rig, (b) Photo of experimental rig

Table 3: Components of the experimental rig

\begin{tabular}{llllll}
\hline \hline No. & Component & No. & Component & No. & Component \\
\hline 1 & load frame & 2 & bearing support & 3 & hoist frame \\
4 & electro-pneumatic coupling & 5 & guide blocks & 6 & guide rails \\
7 & servomotors & 8 & ball screw & 9 & threaded spindle \\
10 & addditional payloads & 11 & displacement sensor & 12 & 3 -axial force sensor \\
13 & 1-axial force sensor & & & & \\
\hline
\end{tabular}
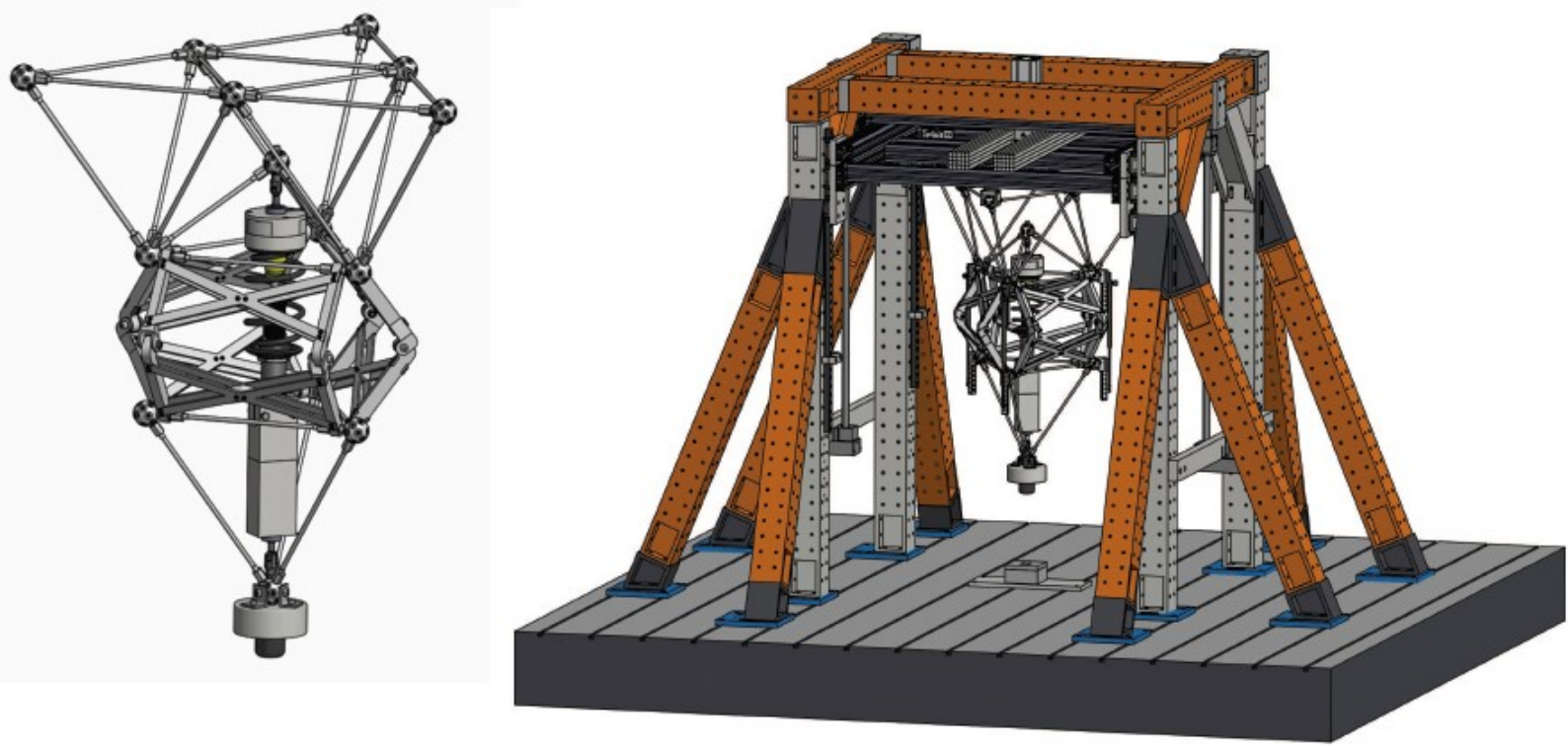

Fig. 6: Perspective CAD illustration of (a) MAFDS and (b) experimental rig

The experimental compression $z_{\mathrm{r}, \mathrm{e}, \mathrm{max}}$ of the MAFDS is recorded by the displacement sensor 11 in Fig. 5 (a) that is based on the linear displacement variable transformer (LVDT) principle. The total load exerted $F_{\text {ef,e,max }}$ by the MAFDS on the ground is recorded by the three-axial strain gauge force 
sensor 12. Similarly, the force $F_{\text {sd,e,max }}$ exerted by the spring damper component of the MAFDS is recorded by the 1-axial strain gauge force sensor 13 .

The experimental drop tests are designed using a simple full factorial (FF) design by varying the mass of the upper structure $m_{\mathrm{u}}$ and the drop height $h$ of the experimental MAFDS, [29]. The FF design takes into account the combinations within a certain range and amount of tests of independent variables and the order of experimental test of these combinations are randomized to avoid systematic errors. In this approach, the significance of the two independent variables $m_{\mathrm{u}}$ and $h$, each having three different values, is considered, see Tab. 4 . Fig. 7 shows the $3 \times 3$ FF design of experiments using $m_{\mathrm{u}}$ and $h$ as varying input variables.

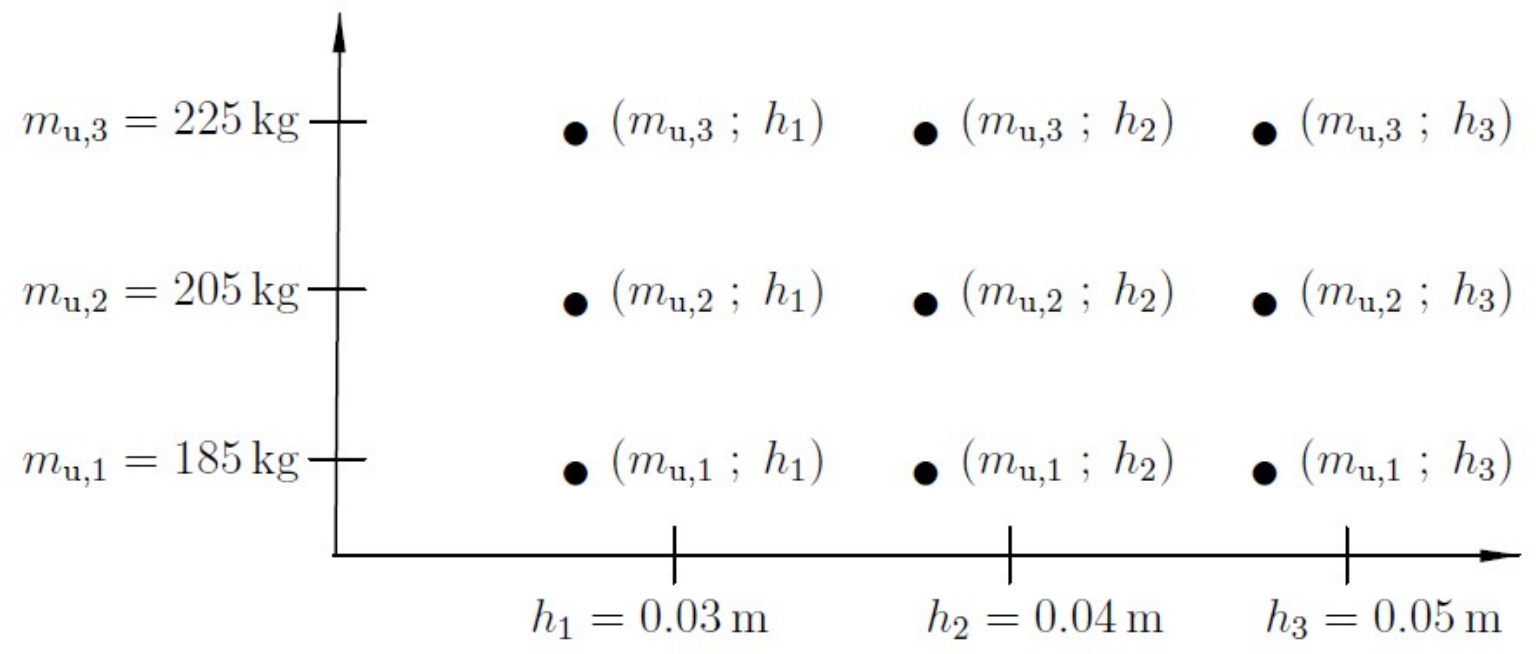

Fig. 7: $3^{2}$ FF design of experiments on MAFDS

This design takes into account both the main effects and the interaction effects of the two variables $m_{\mathrm{u}}$ and $h$. The main effect considers the influence of one independent variable on the output vector $\boldsymbol{y}_{\mathrm{e}, \max }$, while ignoring the influence of other independent variables. In Fig. 7, the experimental mass $m_{\mathrm{u}}$ varies as per the combinations of $\left(m_{\mathrm{u}, 1} ; h_{1}\right),\left(m_{\mathrm{u}, 2} ; h_{1}\right)$ and $\left(m_{\mathrm{u}, 3} ; h_{1}\right)$, while the experimental drop height $h$ remains constant. In this case, the variation in the experimental output $\boldsymbol{y}_{\mathrm{e}, \max }$ is influenced by single variable $m_{\mathrm{u}}$. Conversely, an interaction effect of the independent variable occurs when its influence on the output relies on the value of the other independent variable also. For example, in the combinations $\left(m_{\mathrm{u}, 2} ; h_{3}\right)$ and $\left(m_{\mathrm{u}, 3} ; h_{2}\right)$ the output $\boldsymbol{y}_{\mathrm{e}, \max }$ is influenced by both $m_{\mathrm{u}}$ and $h$.

\section{Quantification of Model Uncertainty}

The four different simplified 2DOF mathematical models a) to d) introduced in section predict the dynamic response differently. The resulting model uncertainty is quantified with the BAYESIAN intervalbased hypothesis testing approach [20], [30]. This approach includes the uncertainty in the mathematical model prediction due to uncertain model parameters and the uncertainty in the experimental observations due to measurement variability.

\subsection{Bayesian Interval Hypothesis-Based Method}

This chapter gives an overview of the Bayesian interval-based approach to quantify the model uncertainty in the mathematical models. The first step is to estimate the difference data matrix $\boldsymbol{Y}_{\mathrm{d}}$ between the model simulation data matrix $\boldsymbol{Y}_{\mathrm{m} \text {,max }}$ and the experimental data matrix $\boldsymbol{Y}_{\mathrm{e} \text {,max }}$. The difference data matrix $\boldsymbol{Y}_{\mathrm{d}}$ consists of difference vectors $\boldsymbol{y}_{d} \in \mathbb{R}^{1 \times P}$, which is tested under the Bayesian interval based hypothesis approach. The number of columns $P$ of the difference data matrix $\boldsymbol{Y}_{\mathrm{d}}$ represents the number of output variables which is equal to 3 , according to (3). The null hypothesis $H_{0}$ occurs when the difference vector $\boldsymbol{y}_{d}$ falls within the acceptable threshold limits $\varepsilon$. Conversely, the alternate hypothesis 
occurs when the difference vector $\boldsymbol{y}_{d}$ falls outside the acceptable threshold limits $\varepsilon$. The Bayes factor $B$ is computed by estimating the probability of the occurrence of the null hypothesis $p\left(\boldsymbol{y}_{d} \mid H_{0}\right)$ to the probability of occurrence of the alternate hypothesis $p\left(\boldsymbol{y}_{d} \mid H_{1}\right)$.

\subsubsection{Random Difference Row Vector}

Fig. 8 summarises the Bayesian interval approach to, first, derive the random difference row vector $\boldsymbol{y}_{\mathrm{d}}$.

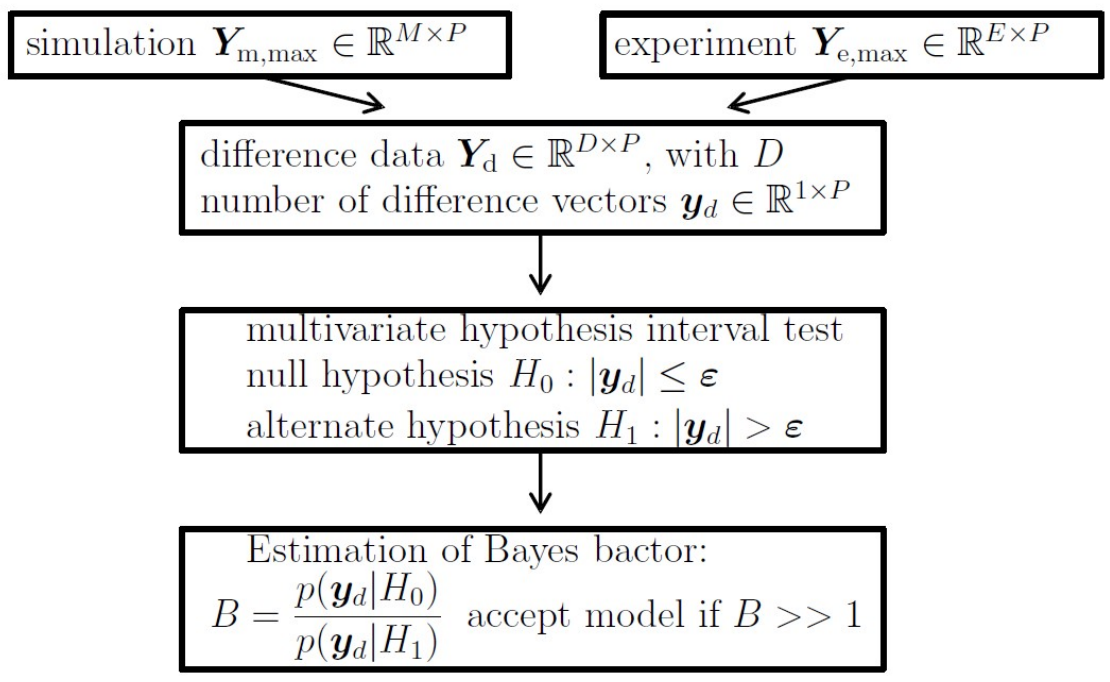

Fig. 8: Bayesian interval based hypothesis approach

Considering that the mathematical models predict the non-deterministic simulation data matrix $\boldsymbol{Y}_{\mathrm{m}, \max } \in \mathbb{R}^{M \times P}$ where $M$ is the number of simulation samples and $P$ is the number of components in the output vector $\boldsymbol{y}_{\mathrm{m} \text {,max }}$ in (3). The number of simulation samples $M$ can be determined using the convergence criteria, for example $M=5.000$ samples. Using the MonTE CARLO method, the uncertainty in the model parameters is propagated through the mathematical models, thereby resulting in non-deterministic output data $\boldsymbol{Y}_{\mathrm{m}, \max }$. Each row vector of $\boldsymbol{Y}_{\mathrm{m}, \max }$ is represented as $\boldsymbol{y}_{\mathrm{m}, \max } \in \mathbb{R}^{1 \times P}$, where $m=1,2,3, \ldots, M$. The model parameters under uncertainty quantification are the stiffness $k$, damping $b$ of the spring-damper as well as the stiffness $k_{\text {ef }}$ of the elastic foot of the MAFDS. It is to be noted that the governing functional relations in Tab. 1 remain deterministic. From the experimental drop tests of MAFDS, the experimental data matrix $\boldsymbol{Y}_{\mathrm{e}, \max } \in \mathbb{R}^{E \times P}$ is obtained, where $E=10$ is the number of repetition of experiments. Each row vector of $\boldsymbol{Y}_{\mathrm{e}, \max }$ is represented as $\boldsymbol{y}_{\mathrm{e}, \max } \in \mathbb{R}^{1 \times P}$, where $e=1,2,3, \ldots, E$. The number of rows of the matrix $\boldsymbol{Y}_{\mathrm{e}, \max }$ is the number of repetitions of the experiments without changing the input variables. Tab. 4 lists the input variables that are used for validation. The number of columns of $\boldsymbol{Y}_{\mathrm{e}, \max }$ remain same as that of $\boldsymbol{Y}_{\mathrm{m} \text {,max }}$, since the experimental output vector $\boldsymbol{y}_{\mathrm{e}, \max }$ in (20) and the simulation output vector $\boldsymbol{y}_{\mathrm{m}, \max }$ in (3) have similar number of components.

Table 4: FF design of experiments with experimental upper mass $m_{\mathrm{u}}$ and the experimental drop height $h$

\begin{tabular}{ccccc}
\hline Variable level & Mass variable $m_{\mathrm{u}}$ & Value in kg & Drop height variable $h$ & Value in $\mathrm{m}$ \\
\hline \hline 1 & $m_{\mathrm{u}, 1}$ & 185 & $h_{1}$ & 0.03 \\
2 & $m_{\mathrm{u}, 2}$ & 205 & $h_{2}$ & 0.04 \\
3 & $m_{\mathrm{u}, 3}$ & 225 & $h_{3}$ & 0.05 \\
\hline
\end{tabular}


The model simulation as well as the experimental data matrices are represented as

$$
\boldsymbol{Y}_{\mathrm{m}, \max }=\left[\begin{array}{ccc}
z_{\mathrm{r}, \mathrm{m}, \max , 1} & F_{\mathrm{sd}, \mathrm{m}, \max , 1} & F_{\mathrm{ef}, \mathrm{m}, \max , 1} \\
z_{\mathrm{r}, \mathrm{m}, \max , 2} & F_{\mathrm{sd}, \mathrm{m}, \max , 2} & F_{\mathrm{ef}, \mathrm{m}, \max , 2} \\
\vdots & \vdots & \vdots \\
z_{\mathrm{r}, \mathrm{m}, \max , M} & F_{\mathrm{sd}, \mathrm{m}, \max , M} & F_{\mathrm{ef}, \mathrm{m}, \max , M}
\end{array}\right] ; \boldsymbol{Y}_{\mathrm{e}, \max }=\left[\begin{array}{ccc}
z_{\mathrm{r}, \mathrm{e}, \max , 1} & F_{\mathrm{sd}, \mathrm{e}, \max , 1} & F_{\mathrm{ef}, \mathrm{e}, \max , 1} \\
z_{\mathrm{r}, \mathrm{e}, \max , 2} & F_{\mathrm{sd}, \mathrm{e}, \max , 2} & F_{\mathrm{ef}, \mathrm{e}, \max , 2} \\
\vdots & \vdots & \vdots \\
z_{\mathrm{r}, \mathrm{e}, \max , E} & F_{\mathrm{sd}, \mathrm{e}, \max , E} & F_{\mathrm{ef}, \mathrm{e}, \max , E}
\end{array}\right] .
$$

The next step is to estimate the difference matrix $\boldsymbol{Y}_{\mathrm{d}} \in \mathbb{R}^{D \times P}$ between the simulation data matrix $\boldsymbol{Y}_{\mathrm{m}, \max }$ and the experimental data matrix $\boldsymbol{Y}_{\mathrm{e}, \max }$. The rows of the difference matrix $\boldsymbol{Y}_{\mathrm{d}}$ consist of the difference vector $\boldsymbol{y}_{d} \in \mathbb{R}^{1 \times P}$. It is computed by taking the row vector difference the between $\boldsymbol{y}_{\mathrm{m}, \max }$ and $\boldsymbol{y}_{\mathrm{e}, \max }$. Hence, the number of rows of $\boldsymbol{Y}_{\mathrm{d}}$ is obtained as $D=M \cdot E$. The difference vector $\boldsymbol{y}_{d} \in \mathbb{R}^{1 \times P}$ in (22) is tested under the BAYESIAN interval based hypothesis approach for $d=1,2,3, \ldots, D$.

$$
\boldsymbol{y}_{d}=\boldsymbol{y}_{\mathrm{m}, \max }-\boldsymbol{y}_{\mathrm{e}, \max }
$$

where the difference index

$$
d=1+\mathrm{E}(m-1)-(1-e) .
$$

The difference vector $\boldsymbol{y}_{d}$ is assumed to follow a discrete Gaussian distribution with a mean vector $\mu_{d} \in \mathbb{R}^{1 \times P}$ and a covariance matrix $\Sigma_{\mathrm{d}} \in \mathbb{R}^{P \times P}$. The reason for assuming a Gaussian distribution is due to the fact that also the randomly picked samples $\boldsymbol{y}_{\mathrm{m}, \max }$ and $\boldsymbol{y}_{\mathrm{e}, \max }$ for the simulation and the experiment are assumed to follow a Gaussian distribution in these first investigations. However, sampling according to other distributions like e. g. uniform, Weibull or exponential distributions could be an option and are subject to further investigations.

\subsubsection{Difference Row Vector under Null or Alternate Hypothesis}

In section, the non-deterministic difference vector $\boldsymbol{y}_{d}$ is defined in (22). Under the framework of the Bayesian interval hypothesis approach, the null hypothesis $H_{0}$ is represented as $H_{0}:\left|\boldsymbol{y}_{d}\right| \leq \varepsilon$, where $\varepsilon$ is the threshold vector [20], [30]. The threshold vector $\varepsilon$ is defined as

$$
\varepsilon=b_{\text {th }}\left\|\boldsymbol{\mu}_{\mathrm{e}}\right\|
$$

where $\boldsymbol{\mu}_{\mathrm{e}}$ is the mean of the experimental output vector $\boldsymbol{y}_{e}$ and $b_{\mathrm{th}}$ is the threshold coefficient that is to be defined by an expert or a model user [9]. Conversely, the alternate hypothesis is defined as $H_{1}:\left|\boldsymbol{y}_{d}\right|>\varepsilon$. The subsequent subsection defines the likelihoods of observing the null hypothesis $H_{0}$ and the alternate hypothesis $H_{1}$.

The likelihood of null hypothesis

$$
p\left(\boldsymbol{y}_{d} \mid H_{0}\right)=\sum_{d=1}^{\mathrm{D}} \frac{\left|\boldsymbol{\Sigma}_{\mathrm{d}}\right|^{-1 / 2}}{(2 \pi)^{n_{\mathrm{d}} / 2}} e^{\left[-\frac{1}{2} \boldsymbol{y}_{d}^{T} \boldsymbol{\Sigma}_{\mathrm{d}}^{-1} \boldsymbol{y}_{d}\right]}, \text { if } \quad\left|\boldsymbol{y}_{d}\right| \leq \boldsymbol{\varepsilon},
$$

is the probability of the non-deterministic difference vector $\boldsymbol{y}_{d}$ falling within the the threshold limits $[-\varepsilon, \varepsilon]$. The covariance matrix $\Sigma_{\mathrm{d}} \in \mathbb{R}^{P \times P}$ is computed from the difference matrix $\boldsymbol{Y}_{\mathrm{d}}$. Conversely, the likelihood of the alternate hypothesis $H_{1}$ is

$$
p\left(\boldsymbol{y}_{d} \mid H_{1}\right)=\sum_{d=1}^{\mathrm{D}} \frac{\left|\boldsymbol{\Sigma}_{\mathrm{d}}\right|^{-1 / 2}}{(2 \pi)^{n_{\mathrm{d}} / 2}} e^{\left[-\frac{1}{2} \boldsymbol{y}_{d}^{T} \boldsymbol{\Sigma}_{\mathrm{d}}^{-1} \boldsymbol{y}_{d}\right]}, \text { if }\left|\boldsymbol{y}_{d}\right|>\boldsymbol{\varepsilon},
$$




\subsubsection{Estimation of Bayes factor B}

According to the Bayes theorem, the bayes factor

$$
B=\frac{p\left(\boldsymbol{y}_{d} \mid H_{0}\right)}{p\left(\boldsymbol{y}_{d} \mid H_{1}\right)}
$$

is the ratio of the the difference vector $\boldsymbol{y}_{d}$ falling under the null hypothesis $H_{0}$ and the alternate hypothesis $H_{1}$ [31]. The Bayes factor is dependent on the prior assumptions of the model parameters $k$, $b$ and $k_{\text {ef }}$ as well as on the threshold interval $[-\varepsilon, \varepsilon]$.

As per the authors in [12], the model confidence $M$ based on the Bayes factor $B$ is determined as

$$
M=\frac{B}{B+1} .
$$

\subsection{Model Confidence for Models a) to d)}

In this section the effect of the threshold vector $\varepsilon(24)$ on the model confidence $M(28)$ is discussed. The Bayes factor $B(27)$ as well as its corresponding model confidence $M$ are computed for all the four mathematical models a) to d). Fig. 9 illustrates the effect of variation of the threshold coefficient $b_{\text {th }}$ on the variation of the model confidence $M$ for all the mathematical models a) to d). It is seen that the model $b$ ) with nonlinear stiffness and linear viscous damping shows lower model confidence compared to the models a), c) and d). For example, model b) has $M=80 \%$ for a threshold of around $47 \%$, whereas models a), c) and d) approach $80 \%$ confidence for lower threshold values. The models c) and d) show similar variation of $M$ with respect to the increasing value of $b_{\mathrm{th}}$. This implies that the nonlinear damping assumption in both cases c) and d) seems more adequate than the linear assumptions in a) and b) - regardless of linear or nonlinear stiffness assumption, and leads to higher confidence. Using (28), the model confidence approaches a value of $100 \%$ when the Bayes factor $B$ approaches $\infty$. Conversely, for a value of $B=1$, the corresponding $M=50 \%$.

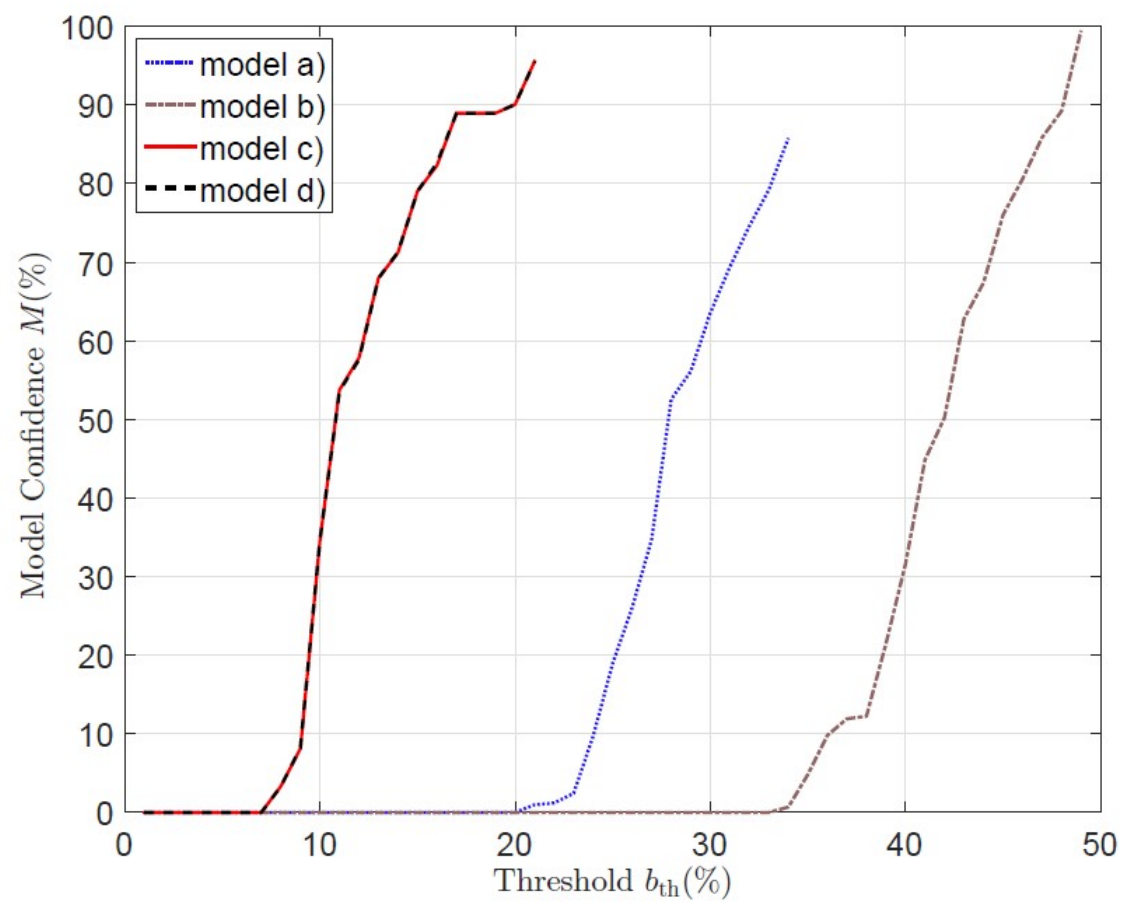

Fig. 9: Model confidence $M$ based on increasing threshold coefficient $b_{\text {th }}$ 


\section{Conclusion and Outlook}

In this contribution, model uncertainty in a two degree of freedom mathematical model that is represented using four different functional relations is quantified via BAYESIAN interval based hypothesis approach. The four different models are based on linear, nonlinear, axiomatic and empiric assumptions. The BAYES factor and its corresponding model confidence are used to quantify the model uncertainty. It is found that the mathematical models with empiric nonlinear damping show higher model confidence compared to the models with linear damping assumptions, regardless of linear or nonlinear stiffness assumption. In future work, the authors explore the possibility of including the uncertainty about the functional relations of the simple two degree of freedom mathematical model vs. a more complex finite element model under the framework of MARKov CHAIN MONTE CARLO simulation.

\section{Acknowledgements}

The authors thank the German Research Foundation DFG for funding this research within the SFB 805 .

\section{References}

[1] Oberkampf, W., Timothy, T., "Verification and Validation in Computational Fluid Dynamics", Progress in Aerospace Sciences, 2002, pp. 209-272.

[2] Oberkampf, W., DeLand, S., Rutherford, B., Diegert, K., Alvin, K., "Error and uncertainty in modelling and simulation", Reliability Engineering and System Safety, 2002, pp. 333-357.

[3] Kennedy, M., O’Hagan, A., "Bayesian Calibration of Computer Models", Journal of the Royal Statistical Society, Vol. 63, No. 3, 2001, pp.425-464.

[4] Rebba, R., Huang, S., Liu, Y., Mahadevan, S.,"Statistical validation of simulation models", Int. J. Materials and Product Technology, Vol. 25, 2006.

[5] Liu, Y., Chen, W., Arendt, P., Huang, H., "Toward a better understanding of model validation metrics", Journal of Mechanical Design, Vol.133, 2011.

[6] Zhao, L., Lu, Z., Yun, W., Wang, W., "Validation metric based on Mahalanobis distance for models with multiple correlated responses", Reliability Engineering and System Safety, 2017, pp. 80-89.

[7] Roy, C., Oberkampf, W., "A comprehensive framework for verification, validation, and uncertainty quantification in scientific computing", Comput. Methods Appl. Mech. Engrg., Orlando, 2011, pp. 2131-2144.

[8] ASME, "Guide for Verification and Validation in Computational Solid Mechanics", ASME Standard 10-2006, New York, 2006.

[9] Zhan Z., Fu, Y., Jang, R.,'Bayesian based multivariate model validation method under uncertainty for dynamic systems", Journal of Mechanical Design, Vol. 134, 2012.

[10] Mahadevan, S.,'Uncertainty quantification for Decision-Making in Engineered Systems", In: Proceedings of the International Symposium on Engineering under Uncertainty: Safety Assessment and Management, 2013

[11] Ling, Y., Sankaraman, S.,"Quantitative model validation techniques: New insights", Reliability Engineering and System Safety, 2013, pp. 217-231.

[12] Sankararaman, S., Mahadevan, S., "Model validation under epistemic uncertainty", Reliability Engineering and System Safety, 2011, pp. 1232-1241.

[13] Sankararaman, S., Ling, Y., Mahadevan, S., "Uncertainty quantification and model validation of fatigue crack growth prediction", Reliability Engineering and System Safety, 2011, pp. 14871504. 
[14] Mahadevan, S., Rebba, R.,"Validation of reliability computational models using Bayes networks", Reliability Engineering and System Safety, 2011, pp. 223-232.

[15] Jiang, X., Mahadevan, S.,'Bayesian validation assessment of multivariate computational models", Journal of Applied Statistics, Vol. 35, 2008, pp. 45-65,

[16] Zhan, Z., Fu, Y., Yang, R., "An Enhanced Bayesian based model validation method for dynamic systems", Journal of Mechanical Design, Vol. 133, 2011.

[17] Platz, R., Ondoua, S., Habermehl, K., Bedarff, T., Hauer, T., Schmitt, S., Hanselka, H.: Approach to validate the influences of uncertainties in manufacturing on using load-carrying structures, USD 2010 International Conference on Uncertainty in Structural Dynamics, 20-22 Sep., Leuven, pp. 5319-5333, 2010.

[18] Melzer, C., Platz, R., Melz, T., "Comparison of Methodical Approaches to Describe and Evaluate Uncertainty in the Load-Bearing Capacity of a Truss Structure", Fourth International Conference on Soft Computing Technology in Civil, Structural and Environmental Engineering, Civil-Comp Press, Stirlingshire, United Kingdom, paper 26, 2015.

[19] Li, S. and Platz, R. (2017), "Observations by Evaluating the Uncertainty of Stress Distribution in Truss Structures Based on Probabilistic and Possibilistic Methods", ASME Journal of Verification, Validation and Uncertainty Quantification, 2(3):031006-031006-9, 2017.

[20] Jiang, X., Mahadevan, S., "Bayesian wavelet method for multivariate model assessment of dynamic systems", Journal of Sound and Vibration, 2007.

[21] Enss, G., Gehb, C., Götz, B.; Melz, T., Ondoua, S., Platz, R., Schäffner, M., Load transferring device, Patent DE102014106858.A1, 2015.

[22] Schaeffner, M., Goetz, B., Platz, R., "Active buckling control of a beam-column with circular cross-section using piezoelastic supports and integral LQR control”, Journal of Smart Materials and Structures, 2016.

[23] Gehb, C. ,Platz, R., Melz, T., "Active load path adaption in a simple kinematic load-bearing structure due to stiffness change in the structure supports", Journal of Physics, Conference Series 744, 2016.

[24] Goetz, B., Schaeffner, M., Platz, R., Melz, T.: "Lateral vibration attenuation of a beam with circular cross-section by a support with integrated piezoelectric transducers shunted to negative capacitances", Journal of Smart Materials and Structures, 2016.

[25] Enss, G., Platz, R., "Evaluation of uncertainty in experimental active buckling control of a slender beam-column using Weibull analysis", Journal of Mechanical Systems and Signal Processing, 2016.

[26] Schaeffner, M., Platz, R., "Gain-Scheduled $H_{\infty}$ Buckling control of a Circular Beam-Column Subject to Time-Varying Axial Loads", Smart Materials and Structures (IOP Publishing Ltd), 27(6), 065009, 2018.

[27] Goetz, B. , Platz, R. and Melz, T. (2018), "Effect of static axial loads on the lateral vibration attenuation of a beam with piezo-elastic supports", Smart Materials and Structures (IOP Publishing Ltd), 27(3), 035011, 2018.

[28] Goetz, B., Platz, R. and Melz, T. (2017), "Consistent Approach to Describe and Evaluate Uncertainty in Vibration Attenuation using Resonant Piezoelectric Shunting and Tuned Mass Dampers", Mechanics and Industry (EDP Sciences), Vol. 18, Nr. 108, (15p), 2017.

[29] Dean, A., Voss, D. "Design and Analysis of Experiments", Springer Verlag New York, 1999.

[30] Pan, H., Castanier, M., "Model Validation for simulations of vehicle systems", In: Proccedings of MSTV Mini Symposium, Michigan, 2012.

[31] Winkler, R.: "Introduction to Bayesian Inference and Decision", Holt, Rinehart and Winston Inc., 1972. 